\title{
Género y migración internacional en la experiencia latinoamericana. De la visibilización del campo a una presencia selectiva
}

\author{
Gender and international migration in latin-american experience. \\ From visibilization of the area to the selective presence
}

\author{
Gioconda Herrera \\ FLACSO Ecuador \\ gherrera@flacso.org.ec
}

Recibido: 16.06 .2011

Aprobado definitivamente: 13.12 .2011

\section{RESUMEN}

En los últimos diez años la producción de trabajos sobre género y migración ha crecido enormemente, aunque muchos de estos trabajos signifiquen, sobre todo, el abordaje de la experiencia de las mujeres en los procesos migratorios. Ya no podemos entonces hablar de la invisibilidad del tema, tal como se planteaba en los balances efectuados hasta hace diez o quince años. Este artículo pretende plantear tres ideas sobre la presencia selectiva de los estudios sobre migración y género partiendo de las investigaciones sobre migración ecuatoriana. La primera idea es que esta relación entre género y migración no es tan nueva y que debemos revisitar los viejos debates sobre género y transformaciones de la estructura agraria que se produjeron en América Latina y, principalmente, en la región andina en los años 1970 y 1980 para entender los cambios ocurridos con la migración internacional, sobre todo, en las experiencias de circuitos migratorios en donde se enlazan procesos de migración interna y externa. La segunda idea consiste en señalar que un paso fundamental observado en los estudios sobre género y migración es el giro de una mirada de la experiencia femenina a intentos por analizar el carácter generizado de las instituciones y los procesos migratorios: el mercado laboral, las políticas migratorias, las estrategias de reproducción familiar, entre otros. Por último, la tercera idea consiste en señalar los aportes desde los estudios de género para repensar la familia -la familia transnacional, la maternidad transnacional, la paternidad transnacional, las infancias transnacionales. En este punto, me parece que la migración internacional es una instancia estratégica para el análisis de la institución de la familia en las sociedades globales y que la perspectiva transnacional posibilita superar miradas rígidas sobre el cambio social.

Palabras clave: género, migraciones, familias transnacionales, cadenas globales de cuidado. 


\begin{abstract}
In the last ten years the academic production on gender and migration has grown enormously, although many of this production signify, above all, the approach to the experience of women in migration processes. No longer can we speak of the invisibility of the issue as raised in the reviews carried up ten or fifteen years ago. This paper intends to develop three ideas about the presence of selective migration and gender studies based on the research on migration in Ecuador. The first idea is that this relationship between gender and migration is not new and that we should revisit old debates about gender and agrarian structure transformations that occurred in Latin America, mainly in the Andean region in the years 1970 and 1980 to understand the changes in the international migration, particularly on the experiences of migrant circuits where processes related internal and external migration. The second idea is to point out that a fundamental step observed in studies on gender and migration is turning a look of women's experience with attempts to analyze the gendered character of migration processes and institutions: the labor market, migration policies, family reproductive strategies, among others. Finally, the third idea is to identify the contributions from gender studies to rethink the family, the family crime, transnational motherhood, fatherhood, crime, transnational childhoods. At this point, I think that international migration is a strategic body for analysis of family institution in global partnerships and that transnational perspective enables us to go beyond rigid interpretations of social change.
\end{abstract}

KEYwords: gender, migration, transnational families, Global Care Chains.

\title{
SUMARIO
}

1. Revisitando los estudios de género en el marco de las transformaciones agrarias de 1970 y 1980 en América Latina. 2. Género, migración latinoamericana y globalización. 3. ¿Las familias transnacionales siguen siendo homogéneas?

\section{SUMMARY}

1. Revisiting gender studies within the framework of the agrarian reforms of 1970s and 1980s in Latin America. 2. Gender, migration and globalization in Latin America. 3. Do transnational families remain homogeneous? 4. Conclusions. 
Al igual que todo campo de conocimiento en formación el análisis de género en la migración internacional en América Latina ha abordado temáticas de manera selectiva, privilegiando unas problemáticas y excluyendo otras, priorizando ciertos espacios y ámbitos de estudio por encima de otros y analizando ciertas relaciones de género más que otras. Esto puede explicarse por varios factores: tiene que ver con el itinerario de los propios estudios sobre migración en el continente, con lo se ha denominado las políticas del lugar y con una mayor o menor legitimidad de los estudios feministas en los espacios de producción académica. En todo caso, es innegable que en los últimos diez años la producción de trabajos sobre género y migración ha crecido enormemente, aunque muchos de estos trabajos signifiquen sobre todo el abordaje de la experiencia de las mujeres en los procesos migratorios. Ya no podemos entonces hablar de la invisibilidad del tema, más bien la reflexión debe partir reconociendo su presencia cada vez mayor en el campo de los estudios migratorios y mirar de qué manera se ha ido construyendo esa presencia. ¿Qué temas se han privilegiado en el análisis de género de la migración internacional? ¿De qué manera han evolucionado estos temas a lo largo de los últimos veinte años? ¿Qué momento y actor del proyecto migratorio son los que han prevalecido en los análisis de género? ¿La partida? ¿La llegada? ¿Los y las que se quedan? ¿Los y las que se van? ¿De qué manera influyen las construcciones sociales de género en la construcción de vínculos transnacionales? Todas estas preguntas pueden orientar un análisis que capte la presencia selectiva del género en la reflexión sobre los procesos migratorios internacionales.

En este artículo examino esta presencia selectiva del género en los estudios sobre migración partiendo de las investigaciones que más conozco, aquellas sobre migración latinoamericana, para colocar sobre la mesa de discusión tres ideas: En primer lugar, que esta relación entre género y migración internacional no es tan nueva y que debemos revisitar los viejos debates sobre género y transformaciones de la estructura agraria que se produjeron en América
Latina y principalmente en la región andina en los años 1970 y 1980 para entender los cambios ocurridos con la migración internacional, sobre todo en las experiencias de circuitos migratorios en donde se enlazan procesos de migración interna y externa. Esto permite entender que la migración internacional no se instala como una experiencia totalmente nueva sobre una realidad de género fija sino que más bien es parte de procesos sociales, históricos, culturales más amplios.

En segundo lugar, un paso fundamental que se observa en los estudios sobre género y migración es el giro de una mirada de la experiencia femenina a intentos por analizar el carácter sexualmente construido de las instituciones y los procesos migratorios. Así, se empieza a analizar al mercado laboral y su segmentación por sexo o los impactos diferenciados sobre hombres y mujeres de las políticas migratorias o el papel de mujeres y varones en las estrategias de reproducción social de las familias, entre otros. En este giro ha sido muy importante el aporte de teóricas que han reflexionado sobre la relación entre género y globalización, mostrando cómo los procesos de globalización económica están moldeados por relaciones de desigualdad de género a nivel estructural (Sassen, 2003; Bakker y Gil, 2003). Esta mirada ha privilegiado ciertos ámbitos de estudio por sobre otros: por ejemplo el trabajo doméstico ejercido por mujeres inmigrantes ha recibido enorme atención en los últimos años, invisibilizando otro tipo de actividades ejercidas por mujeres migrantes, tales como el trabajo agrícola o en manufacturas y sus conexiones con cadenas productivas globales ${ }^{1} \mathrm{o}$ el trabajo profesional de mujeres migrantes. Pero además, el propio trabajo de cuidado ha sido predominantemente examinado de manera homogénea sin tomar en cuenta a otros actores dentro de la propia economía del cuidado como los varones en calidad de cuidadores, las enfermeras o los y las cuidadoras en origen. Por tanto, en el análisis de la relación entre género, globalización y migración latinoamericana, se produce una presencia selectiva de ciertas mujeres migrantes en el análisis en detrimento de otros sujetos y otras desigualdades que tienen que ver con diferencias

\footnotetext{
${ }^{1}$ Ver por ejemplo los trabajo de Fernandez Kelly y García (1990) sobre las mujeres migrantes en los talleres textiles o el trabajo de Barndt (2002) sobre el trabajo de las mujeres en las cadena del tomate entre México y Estados Unidos, o el de la castaña para exportación en el caso de la población de Rivera Alta en Bolivia de Montero y Poveda (2003).
} 
intergeneracionales, masculinidades subordinadas, jerarquías entre mujeres.

En tercer lugar, un tema especialmente presente en la literatura sobre género y migración en América Latina se deriva de las reflexiones en torno a las familias migrantes y especialmente en torno a "las que se quedan". Se ha producido un revivir de los estudios sobre familia desde el feminismo que examina los cambios y continuidades en la conformación y reproducción de los lazos familiares en la experiencia migratoria. En este articulo examino los aportes desde los estudios de género para repensar la familia - la familia transnacional, la maternidad transnacional, la paternidad transnacional, las infancias transnacionales - En este punto me parece que la migración internacional es una instancia estratégica para el análisis de la institución familia en las sociedades globales y que la perspectiva transnacional posibilita superar miradas rígidas sobre el cambio social.

\section{REVISITANDO LOS ESTUDIOS DE GÉNERO EN EL MARCO DE LAS TRANSFORMACIONES AGRARIAS DE 1970 Y 1980 EN AMÉRICA LATINA}

En esta sección intento ubicar continuidades y rupturas entre los conceptos que sirvieron para interpretar los impactos de las migraciones internas sobre las mujeres rurales y aquellos que son ahora utilizados para analizar las migraciones internacionales pues se constata que los estudios sobre mujer rural realizados en ese mismo período anuncian muchas de las problemáticas que desde la perspectiva de género se han analizado en los procesos migratorios. En efecto, varias de las herramientas conceptuales que se utilizaron en la década de 1980 para analizar las migraciones internas, por ejemplo las estrategias de sobrevivencia o la importancia de las redes sociales en la decisión de migrar, vuelven a aparecer en el análisis de los fenómenos de la migración internacional en la globalización. De allí que ciertos cuestionamientos que fueron realizados desde el análisis de género a conceptos como hogares, unidades domésticas y estrategias familiares de supervivencia son todavía bastante útiles para una lectura del fenómenos migratorio. Así mismo, en ciertos análisis sobre el rol de las mujeres en los procesos migratorios internacionales, encontramos ciertas reminiscencias del análisis sobre las mujeres campesinas como íconos de reproducción cultural.

Durante la década los años setenta y ochenta, las migraciones internas fueron motivo de extensos análisis en América Latina pues fueron procesos muy diversos que modificaron profundamente la configuración de los espacios locales y las relaciones sociales y familiares (Ariza, 2000; Carrasco y Lentz, 1985). En efecto, las mujeres latinoamericanas participaron de manera numerosa en los flujos de migración rural urbana que transformaron las ciudades latinoamericanas durante el siglo veinte. Más aún, la migración a las ciudades fue uno de los mecanismos a través de la cuál muchas mujeres ingresaron por primera al mercado laboral, generalmente al sector del trabajo domestico (Ariza, 2000:7).

De acuerdo a la revisión que hacen Carrasco y Lentz hasta mediados de 1980, los estudios sobre migración interna se centraron en analizar los desplazamientos rural-urbanos en el marco de las interpretaciones sobre los procesos de urbanización, las migraciones temporales desde las áreas minifundistas del espacio rural hacia las grandes unidades productivas agrícolas -particularmente a las áreas de plantación- y los movimientos poblacionales de ampliación de la frontera agrícola, es decir los procesos de colonización (Carrasco y Lentz, 1985:7).En un primer momento, los análisis se centraron en lo que se denominó la versión "modernizadora" de la migración. Ésta era analizada como un juego entre factores de expulsión y factores de atracción, que comúnmente se identificaron con variables económicas "objetivas" detrás de las cuáles se perfilaba una dicotomía entre lugar de origen y lugar de destino: se concebían estos espacios como unidades sociales autónomas, la una tradicional y la otra moderna. Esta última atraía mecánicamente al migrante, como imanado por condiciones objetivas favorables. De este marco, se derivaron también interpretaciones sobre los efectos de la migración, entendidos como un conjunto de cambios culturales, actitudes, estilos de vida, que implicaban

\footnotetext{
${ }^{2}$ Esta sección es una versión revisada y actualizada del articulo "Género, familia y migración: lo viejo y lo nuevo" de mi autoría compilado por Norma Fuller (2004).
} 
la desestructuración de las relaciones sociales y de la cultura tradicional. Los conceptos de descomposición, desestructuración, aculturación, asimilación, individualización, fueron centrales en estos trabajos (Carrasco y Lentz, 1985:8).

Si bien estos estudios no mencionaron explícitamente a las mujeres, el supuesto detrás de esta perspectiva era que en estos procesos de cambio las mujeres eran por un lado, portadoras de la matriz cultural tradicional o receptoras pasivas de los embates de la modernidad y que los agentes de cambio eran los varones. Es decir la dicotomía tradición/modernidad se traducía en una oposición mujer/hombre.

Por otro lado, una mirada opuesta a la de la modernización fue aquella que postuló que no se podían analizar los proceso migratorios fuera de la lógica de acumulación capitalista y de la reasignación de los recursos implícita en ésta: la demanda de fuerza de trabajo en el sector industrial y la expansión de los servicios junto con la penetración del capitalismo en el campo, eran los factores estructurales que explicaban la migración campo-ciudad. En esta perspectiva, la migración también es vista como un fenómeno unidireccional de campesinos caminando hacia la proletarización urbana.

Una tercera perspectiva es aquella que postuló que la migración era parte de un conjunto de estrategias de supervivencia de las familias campesinas. Este concepto proponía entender a la migración como una estrategia a la que las familias acudían para resistir a los embates económicos del capitalismo y garantizar la reproducción de las unidades familiares. Este concepto aludía en algunos casos a dinámicas preferentemente económicas (Farell, 1988; Martínez, 1984) otras autoras más bien enfatizaban elementos étnico-culturales (Lentz, 1984). La primera mirada postuló un progresivo desmoronamiento de los mecanismos de solidaridad y reciprocidad de la comunidad y el carácter "erosionador" de la migración (Martínez, 1984); los segundos visibilizaron en los procesos migratorios la conformación de redes y cadenas de apoyo que más bien revivían y reproducían estos mecanismos de solidaridad (Lentz, 1984). Para Lentz, la persona no se lanza al azar a la migración, sino que lo hace en tanto es participa en redes colectivas de información y valorizaciones respecto a ciertos lugares de destino y determinados segmentos del mercado laboral. Con la introducción de este nivel intermedio de análisis -las familias-, empiezan a aparecer las mujeres como actoras económicas importantes, al visualizarse la articulación de sus actividades reproductivas dentro de estas estrategias de supervivencia, con lo productivo. Surgen entonces mujeres articuladas a lógicas familiares que a su vez están conectadas a procesos estructurales. Como veremos más adelante, este argumento vuelve a aparecer con mucha fuerza en los estudios desde la economía política feminista de la globalización.

Más aún, para otros estudios la migración además de integrar una estrategia familiar era también parte de una estrategia comunitaria-campesina de reproducción: unos salen para que otros sigan siendo campesinos y la comunidad sigue siendo el eje de referencia cultural para los que están lejos. Esto lo retoma Geneviéve Cortes (2004) para las migraciones internacionales, en su estudio sobre migración de bolivianos a Argentina que lleva precisamente el nombre de partir para quedarse.

Como vemos, este conjunto de trabajos sobre migraciones internas planteaban ya varias de las perspectivas que serán debatidas en el contexto de las migraciones internacionales en la globalización en los años noventa. Nos referimos a los conceptos de redes sociales y estrategias familiares de supervivencia o de reproducción, entre otros. El interés fundamental de este conjunto de trabajos era matizar la interpretación puramente estructural y económica del fenómeno, colocando a la unidad familiar como un nivel meso de análisis que pudiera rescatar el rol de los agentes sociales, en este caso la familia entendida como un todo uniforme, en la determinación de ciertas dinámicas sociales.

Desde la perspectiva de género se empezó a analizar el impacto de la migración masculina sobre la vida de las mujeres campesinas. La migración es analizada como un factor que altera profundamente la organización social tradicional pero sus consecuencias son variadas. En el caso de una zona rural indígena de Ecuador Rosero (1986) señala el peso del trabajo que recae sobre las hijas mayores y las ancianas, fenómeno que reaparece con el tema de las cadenas globales del cuidado de finales del siglo pasado.

También se señala una mayor valoración social de las mujeres al interior de sus comunidades, debido a su creciente participación en la toma de decisiones frente al trabajo productivo y a la organización 
comunal (Rosero, 1986), tema que será discutido por los primero trabajo que examinan las tensiones entre empoderamiento y control social y sexual de las mujeres cuyos conyugues han emigrado (Borrero y Vega, 1995; Herrera, 2006).

En general, en estos estudios, las migraciones son analizadas como trayectorias individuales masculinas articuladas a estrategias familiares en las cuales las mujeres, las relaciones de género y las diferencias generacionales son tomadas como variables neutras, que se acomodan a esta lógica colectiva pero que no necesariamente son significantes de relaciones de poder y desigualdad. En todo caso lo que interesa señalar es que los fenómenos migratorios internos fueron analizados desde una concepción de la migración que la entendió más allá de la movilidad de personas, como procesos que implicaban intercambios de información, flujos monetarios y materiales, determinadas dinámicas culturales de adaptación y resistencia y la conformación de redes. Por otro lado, hay que señalar igualmente que los esfuerzos estuvieron orientados a analizar cómo estas unidades familiares se articulaban a la economía capitalista precisamente sobre la base de un ordenamiento de género que ocultaba dicha conexión: cómo la reproducción social, el trabajo de las mujeres campesinas subsidiaba la producción capitalista.

\section{GÉNERO, MIGRACIÓN LATINOAMERICANA Y GLOBALIZACIÓN}

Las características de los procesos migratorios desde la región andina de los últimos años provocaron la eclosión de trabajos sobre la feminización de las migraciones y mostraron la pertinencia de fortalecer el análisis de género para una comprensión de las especificidades de los mismos. En efecto, las mujeres empiezan a migrar masivamente ya sea junto a sus conyugues o en procesos de reunificación familiar (principalmente a Estados Unidos), como migrantes independientes, como pioneras de proyectos migratorios familiares o como acompañantes cuidadoras (las abuelas). Es decir, lo hacen desde una multiplicidad de roles e identidades. Estas experiencias van a ser analizadas predominantemente desde dos aristas: sus itinerarios migratorios y procesos de inserción laboral; y los impactos en las familias de las sociedades de origen. A diferencia de México, el Sudeste asiático o las mismas ciudades del norte global, todos lugares en los cuales la relación entre género y globalización es analizada desde la inserción de las mujeres migrantes en la maquila, los talleres textiles o las cadenas globales agrícolas, (por mencionar solo algunos de los nichos laborales globales feminizados), la migración de las mujeres andinas a Europa es examinada de manera preponderante en torno al trabajo doméstico y la economía del cuidado. En cualquier caso, se trate de trabajo agrícola, textil o de cuidado estas experiencias confirman que la segregación ocupacional por sexo de la fuerza de trabajo moldea la demanda de trabajo migrante a nivel global y que los mercados laborales son cada vez más racializados y están estructurados por género tanto en origen como en destino (Sassen, 2003).

Los aportes de los estudios de género en el análisis de la migración andina han ayudado a contrarrestar explicaciones economicistas de la partida, mostrando cómo la decisión de emigrar se produce también como resultado de otro tipo de discriminaciones: étnicas, de género, sexuales. Así, a partir de un análisis centrado en la agencia de las mujeres migrantes, se complementaron las explicaciones económicas con otro tipo de factores como los conflictos familiares (Herrera, 2006) o la violencia doméstica (Camacho, 2009; Román, 2008), la discriminación étnica, o por orientación sexual (Ruiz, 2002). Además de estas diferencias entre hombres y mujeres, se encontraron diferencias generacionales. Los y las jóvenes también veían a la migración como una forma de ampliar sus horizontes de vida y no sólo como un mecanismo de reproducción social y económica de sus familias. En otras palabras, esto permitió cuestionar y complejizar la visión de que la decisión de emigrar era una decisión familiar.

Antes de la feminización de las migraciones a finales del siglo anterior, los análisis de género apuntaban a analizar el impacto de la emigración masculina rural sobre la situación de las mujeres -madres, hijas, esposas- que se quedaban (Borrero y Vega, 1995;). Los estudios señalaban la gran vulnerabilidad de las mujeres campesinas que se quedaban a cargo de sus familias y muchas veces, además de las actividades de cuidado y reproducción debían asumir las tareas productivas agrícolas (Borrero y Vega, 1995). También se señalaba las tensiones que generaba la separación entre esposos y los mecanismos de control 
desplegados por las familias alrededor de la administración de las remesas y el control de la sexualidad de las mujeres (Herrera, 2006). En general, a diferencia de estudios realizados en otros contextos de salida, la migración masculina no parecía influir en procesos de mayor autonomía y empoderamiento de las mujeres, ni tampoco en cambios en la división sexual del trabajo. La investigación de Herrera y Martínez (2002) en la zona rural del sur del Ecuador mostraba cómo los procesos de empoderamiento de las mujeres que se quedaban estaban matizados por aspectos de control de la sexualidad y de falta de decisión frente al uso del dinero remitido.

Una vez sobrevenida la emigración masiva de mujeres, los estudios muestran que las mujeres latinoamericanas en Europa se insertan abrumadoramente en actividades de cuidado, ya sea trabajo doméstico, atención a niños y a adultos mayores y que sus condiciones laborales varían ampliamente dependiendo de su condición migratoria, del acceso a un mercado laboral más formalizado, del tiempo de migración y de su condición familiar (Wagner, 2004; Herrera, 2007; Lagomarsino, 2006; Román, 2009; Camacho, 2009; Hinojosa, 2009;. Muchos trabajos se han centrado en las contradicciones de los procesos migratorios de las mujeres, mostrando que si bien viven procesos de movilidad económica también experimentan procesos de desvalorización social en sus trabajos. Así mismo, se examina que la inserción laboral precaria viene acompañada de grandes vacíos en torno al cuidado que dificultan tanto la reunificación familiar como la organización misma del cuidado una vez que han llevado a sus familias (Herrera, 2008). El carácter mismo del trabajo de cuidado, en el que entran en juego aspectos subjetivos, que van más allá de una prestación de servicios, hablan de contradictorios procesos de dependencia emotiva que se crean entre empleadora y empleada, lo que Ambrosini (2005) denomina familiaridad asimétrica, que contrastan con una independencia y mayor autonomía de las mujeres respecto a sus conyugues, debido al manejo de recursos económicos propios.

La inserción mayoritaria de mujeres en la economía del cuidado en los países de destino ha empezado a ser documentada desde los análisis feministas como parte de un proceso de globalización y privatización de la reproducción social. (Young, 2003; Bakker y Gil, 2003; Ehrenreich y Hochschild, 2002).
La mayoría de estos estudios muestran la articulación existente entre las crisis de reproducción social de los países de origen, causados por recurrentes crisis económicas y sociales, con las crisis de cuidado en los países del norte como factores estructurales que impulsan la migración de las mujeres. Así, autoras como Misra et al. (2005) sostienen que las políticas económicas neoliberales, como los ajustes estructurales, la reestructuración de los Estados de bienestar y las políticas migratorias han contribuido a dar forma a lo que Salazar Parreña denomina la nueva división internacional del trabajo reproductivo (Salazar Parreñas, 2001) y argumentan que procesos laborales como las actividades del cuidado son centrales para una comprensión del funcionamiento de la economía política global (Bakker y Gil, 2003; Misra, et al., 2005). Si el capitalismo siempre ha descansado en una división sexual en la cual las mujeres con su trabajo reproductivo subsidian la economía, las cadenas globales del cuidado estarían expresando este proceso a escala transnacional. En ese sentido, la migración de mujeres estaría mostrando la necesidad de entender el trabajo del cuidado en el marco de las relaciones de género articuladas a las relaciones de clase, étnicas, regionales y geopolíticas.

En efecto, acuñado en 2002 por Hotchschild y Einsenstein en su libro Global Women el concepto de cadenas globales de cuidado empezó a ser utilizado para explicar la migración femenina en varios corredores migratorios en distintas regiones del mundo, y ha servido fundamentalmente para denotar que la migración femenina de los últimos veinte o treinta años se inscribe en un proceso macroestructural de desigualdad global. Es decir, fue más allá de las visiones que hasta entonces habían predominado en la relación entre migración y género, fundamentalmente sobre experiencias diferenciadas entre hombres y mujeres de la experiencia migratoria, o de visiones causales, a veces un poco simplistas, de si las mujeres pierden o ganan con la migración.

Lo que el concepto de cadenas globales de cuidado ha subrayado es que paralelo a los flujos de capitales, de información, de mercancías que circulan en la globalización también se producen circuitos internacionales de cuidadores y especialmente de cuidadoras que garantizan la reproducción social de muchos seres dependientes, especialmente adultos mayores y niños y niñas o discapacitados, y también 
independientes, en diversas partes del planeta. Estos circuitos fueron analizados en la migración de mujeres asiáticas al medio Oriente, a Europa y Estados Unidos primero y más tarde, con el boom se convirtieron en una de las explicaciones más interesantes ala hora de analizar el súbito crecimiento de la migración femenina andina a Europa.

En la revisión que establece Yeats (2005) al concepto se describe a la cadena global de cuidado prototípica con el siguiente ejemplo:

Una hermana mayor de una familia de bajos recursos que cuida de sus hermanas mientras su madre trabaja como niñera cuidando a otro niños cuya madre a migrado y quien, a su vez cuida a los niños de una familia en un país rico. (Yeats, 2005)

La idea básica detrás del concepto es mostrar que mientras más se desciende en la cadena, el valor del trabajo decrece y poco a poco se convierte en no remunerado. Además las transferencias atraviesan ejes de desigualdad complejos que incluyen relaciones de género, clase y raza pero también geopolíticos, intergeneracionales, nacionales, entre otros.

Desde las sociedades de origen, el concepto constituyó una herramienta muy útil para situar en el debate de migración y desarrollo una mirada desde origen; así como los migrantes aportan a través de sus trabajos formales a mantener la seguridad social de los ciudadanos de los países de destino, así mismo las mujeres migrantes, con su trabajo de cuidadoras, aportan también al cuidado de estas otras generaciones del Norte.

También sirvió para entender las estrategias transnacionales de los hogares migrantes, así como las potencialidades y fragilidades de las familias transnacionales. Se trata de un concepto que condensa tanto una perspectiva desde la geografía de las desigualdades pero con un sustrato de género claro, orientado a mostrar que estas transferencias implican desnaturalizar al trabajo doméstico, no remunerado, colocarlo como un eje articulador que explica la razón de ser de estas cadenas a nivel nacional e internacional y por tanto colocar las desigualdades de género como articuladoras de las otras formas de desigualdad.

Si bien fueron muchas las bondades del concepto para entender las matrices de género presentes en esta globalización de la reproducción social en ma- nos de mujeres migrantes, algunos trabajos han empezado a interrogar este concepto debido a ciertos sesgos tales como la idea de "drenajes de cuidado" que parece implicar. En efecto, una mirada detallada a las percepciones y prácticas en torno a los arreglos y negociación de los cuidados implica rescatar, por un lado, la agencia de las mujeres migrantes y por otro lado, las acciones desplegadas por las redes familiares, las redes sociales y el mercado. Pero además, en la configuración de esta organización social del cuidado en la migración se redefinen nociones sobre el cuidado, la maternidad, la identidad de las mujeres migrantes, de las y los cuidadores, y de las personas que demandan cuidados. Es decir, más allá de las transferencias de cuidado, las prácticas que se activan y desactivan con la migración reflejan procesos de desigualdad social al interior de estas redes que no permite captar el concepto de cadenas globales de cuidado.Por ello, interesa también subrayar que las actividades de cuidado no siempre se reparten de manera igualitaria entre las personas, entre las familias, entre hombres y mujeres, entre mujeres de diferentes clases sociales, y no sólo entre países. De ahí que sea necesario complementar esta geografía de la desigualdad global con la reconstrucción de las redes locales de cuidado y la forma cómo éstas están atravesadas por ideologías de género.

\section{3. ¿LAS FAMILIAS TRANSNACIONALES SIGUEN SIENDO HOMOGÉNEAS?}

Un tercer tema que se activa con la salida de las mujeres (y no necesariamente con la salida de los varones) es aquel relacionado con los cambios y continuidades en torno a la familia. En primer lugar se plantea el dilema de si la migración femenina estaría en la raíz de rupturas y procesos de desestructuración familiar o si más bien se asiste a la conformación de otro tipo de familias, las llamadas familias transnacionales, que mantienen lazos materiales y afectivos entre sus miembros, así como mecanismos de toma de decisiones conjuntas sobre el futuro de las familias (Bryceson y Vuorela, 2003; Herrera y Carrillo, 2009). Esta perspectiva sirvió para analizar las nuevas formas de materializar a la familia distante, de fortalecer sus lazos y aquellos con la comunidad y de resaltar la formación selectiva de lazos emocionales y materiales sobre la base de conside- 
raciones espaciales, temporales o relacionadas con necesidades concretas. Análisis en esa línea son los de Sorensen (2008) Herrera y Carrillo (2009) Carrillo (2008) y Reist y Riaño (2008).

¿Cuáles han sido las bondades del concepto de familias trasnacionales desde la perspectiva de género? En primer lugar, uno de los giros más importantes fue el ampliar el marco de análisis de los fenómenos migratorios y mirar a la migración como una práctica social que está presente en el horizonte de vida de las personas que pertenecen a ese campo desde sus distintas posiciones: como migrantes, como cónyuges, desde la posición de hijos/hijas, como integrantes de la misma comunidad, como agentes económicos, políticos. Es decir, involucra y articula de manera simultánea a los que se van pero también a la comunidad de origen y de destino en su conjunto. De esta manera, las mujeres (o los jóvenes) no sólo aparecen en sus distintos papeles de migrantes, más o menos invisibles, sino que pueden ser analizadas fundamentalmente desde un punto de vista relacional respecto a todo el campo (Swetman, 1998). Así la migración, se convierte en un campo social permeado entre otras cosas por desigualdades y jerarquías de género y generacionales. En este marco, se hacen visibles un sinnúmero de actores y procesos que no aparecen en los análisis convencionales de la emigración que toman en cuenta a los migrantes como individuos: el trabajo de las personas que quedan a cargo del cuidado, la sobrecarga de trabajo de algunos de estos actores, como las abuelas y las hermanas mayores, el sentido social y simbólico de las remesas, más allá de su peso macroeconómico, entre otros.

Por otro lado, el análisis de género permitió relativizar las visiones armoniosas en torno a la idea de comunidad y familia transnacional. El trastocar el sentido de presencia física por presencias imaginadas, se alcanzaba por medio no sólo de la información, los intercambios materiales y simbólicos que fluyen a través de las redes, sino también de formas de ejercicio del poder intrafamiliar. Estas redes pueden ser espacios de reproducción de relaciones de poder y desigualdad entre hombres, mujeres, padres/ madres e hijos. Así, por ejemplo para D'Aubeterre (2001) los flujos migratorios pueden conformar un tipo de familia transnacional que no necesariamente rompe con los patrones hegemónicos de la familia, a pesar de que se trastocan muchas de las prácticas co- tidianas. La conyugalidad a distancia, que supone la no corresidencia, las continuas negociaciones entre marido y mujer en la toma de decisiones concernientes a los procesos de producción y reproducción que involucran al grupo doméstico, la fidelidad femenina y la permanencia de los bienes sociales y simbólicos tales como el honor o el prestigio, tienden a ser procesos conflictivos (Priblisky, 2007). Así mismo, como lo han señalado Pierrette Hondagneu-Sotelo y Ávila (2003), esta nueva modalidad de familia implica diversas formas de explotación económica encubiertas por la ideología del parentesco y no conlleva a un cuestionamiento de las representaciones hegemónicas de género. En otras palabras, quedan de lado los juegos de poder que permean las decisiones e intereses de las estrategias familiares. No todos los miembros de la familia actúan en igualdad de condiciones ni cuentan con las mismas capacidades de negociación. En definitiva, el concepto de familia transnacional acentúa el carácter socialmente construido de la familia sin negar la existencia de relaciones de poder a su interior y de procesos selectivos que los distintos agentes emprenden para actuar dentro de sus redes.

No obstante una de las limitaciones de este concepto es que excluye un número importante de situaciones en que la familia no se recrea y más bien existe ruptura, la conformación de otras familias superpuestas y también abandono. Por otro lado, tanto la recuperación de la experiencia de las migrantes, con sus especificidades y diferencias respecto a la trayectoria de los varones y su ubicación analítica en un campo social atravesado por desigualdades de clase, étnicas y de raza, son elementos que permiten pensar los fenómenos de la migración desde la perspectiva diferenciada de los actores involucrados en ella, más allá del concepto homogenizante de la familia. Si bien uno de los aspectos claves en la comprensión de las dinámicas migratorias es el papel que juega la familia, ésta debe ser entendida como locus de soporte social y emocional pero también como un campo conflictivo de circulación de relaciones de poder entre los diferentes miembros que la conforman. Esta visión permite rescatar la diversidad de experiencias entre los distintos miembros de una familia y por tanto puede incluir la visión de los hijos/as de migrantes. En ese punto lo que los estudios muestran es que precisamente se ha avanzado muy poco en mirar las experiencias de los distintos 
miembros de la familia. El trabajo de Jason Pribilsky (2007) sobre la experiencia de los "hombres migrantes en tanto hombres" es una excepción y tal vez uno de los pocos trabajos que profundiza en el tema de la paternidad transnacional en la experiencia de la migración ecuatoriana a Estados Unidos y muestra precisamente que esta paternidad transnacional se construye de manera relacional y contextual. La etnografía de Pribilsky muestra que los hombres migrantes no reproducen identidades masculinas fijas y establecidas sino que emergen nuevas identidades en respuesta a situaciones nuevas y también a los cambios en la posición e identidades de sus conyugues en origen (2007: 19). Así mismo en lugar de asumir que la paternidad transnacional es una identidad fugaz o poco relevante en la experiencia masculina migrante, demuestra que es fundamental en sostener el proyecto migratorio de la familia. Otro trabajo en esta línea es el de Carolina Rosas (2008) quien analiza la construcción de la masculinidad entre familias de migrantes mexicanos a Chicago. No encontramos el mismo tipo de análisis respecto a infancias transnacionales en la migración latinoamericana. Un estudio que explora esta faceta es el de Michelle Gamburg (2005) en Sri Lanka, la autora analiza la reconstrucción que hacen de su experiencia los hijos e hijas de madres migrantes al Medio Oriente y muestra cómo existe un ejercicio de memoria selectiva en estas reconstrucciones.

\section{CONCLUSIÓN}

La revisión del campo de género y migración en la experiencia latinoamericana que está concluyendo no ha pretendido ser exhaustiva. Uno de los temas que no se ha abordado, por ejemplo, es aquel relativo a la trata de mujeres, tema que demandaría un articulo entero debido a la controversia en torno a temas como el derecho al trabajo versus la explotación sexual, todas problemáticas que escapan al sentido de este articulo y que ha conocido cierto desarrollo en los estudios sobre migración latinoamericana a Europa. ${ }^{3}$ Tampoco se ha trabajado a profundidad la relación entre género, Estado y políticas migratorias. Este es unos de los temas a seguir explorando en los estudios para continuar con una línea crítica al carácter patriarcal de las instituciones y los procesos sociales y de esa manera entender mejor cómo instituciones sociales como los estados, los mercados, las familias están moldeadas por ideología de género pero también moldean cotidianamente la vida de hombres y mujeres aquí y al otro lado del itinerario migratorio. Un tercer tema, prácticamente ausente en los estudios reseñados es aquel relativo a la sexualidad. El trabajo de García y Oñate (2008) sobre transexuales ecuatorianos en Murcia, explora una línea que merece la pena seguir trabajando y sobre la que no existen muchos trabajos todavía. La experiencia migratoria de transexuales, transgéneros, gays y lesbianas ha sido todavía poco examinada desde los estudios de género a pesar de ser una comunidad importante de la migración latinoamericana. Quedan entonces todavía varios temas por explorar con el fin de que esta presencia selectiva se traduzca en una mirada inclusiva y más exhaustiva de las desigualdades de género presentes en la experiencia migratoria y su articulación con procesos globales de desigualdad estructural. En ese sentido, el campo de género y migración sigue siendo todavía un campo abierto que busca ser enriquecido, fortalecido y transformado.

${ }^{3}$ Ver por ejemplo los trabajos de Adriana Piscitelli(2008) o de Laura Oso (2008). 


\section{BIBLIOGRAFÍA}

Ambrosini, M. y Torre, A. (a curi di) (2005): Secondo rapporto sull'immigrazione a Génova, Génova, Fratelli Frilli Editori.

Ariza, M. (2000): Ya no soy la que dejé atrás... mujeres migrantes en República Dominicana, México, Instituto de Investigaciones Sociales-Editorial Plaza y Valdés.

Bakker, I., y Gill, S., (2003): Power, Production, and Social Reproduction. Human Insecurity in the Global Political Economy, New York, Palgrave MacMillan.

Barndt, D. (2002): Tangled Routes: Women, Work and Globalization on the Tomato Trail, Ontario, Garamond University Press.

Borrero Vega, A.L. y Vega Ugalde, S. (1995): Mujer y Migración. Alcance de un fenómeno nacional y regional, Quito, Abya Yala.

Bryceson, D. y Vuorela, U. (2001): The Transnational Family New European Frontiers and Global Networks, Oxford, Oxford University Press.

CAmacho, G. (2009): Mujeres Migrantes: trayectoria laboral y perspectiva de desarrollo humano, Quito, CLACSO IEE.

CARrillo, M.C. y Cortés, A. (2008) "Por la migración se llega a Ecuador: una revisión de los estudios sobre la migración ecuatoriana en España.” en Gioconda Herrera y Jacques Ramírez (ed.), América Latina migrante: estado, familias, identidades, Quito, FLACSO - Ecuador / Ministerio de Cultura del Ecuador.

Carrasco, H. y Lentz, C. (1985):Migrantes Campesinos de Licto yFlores, Quito, Abya Yala.

CoRtes G. (2005): Partir para quedarse. Supervivencia y cambios en las sociedades campesinas andinas, La Paz, IFEA-IRD.

D’Aubeterre, M. E. (2001): “¿Todos estamos bien? Género y parentesco en familias de transmigrantes poblanos”, Washington DC, LASA, Septiembre 6-8.

Ehrenreich, B. \& Hochschild, A.R. (2002): Global Woman: Nannies, Maids And Sex Workers in The New Economy, New York, Henry Holt and Company Farrel, G. et al. (1988): Caminantes y Retornos, Quito, Instituto de Estudios Ecuatorianos.

GarcíA, A. \& Oñate, S. (2008) “Transexuales ecuatorianas: el viaje y el cuerpo”, en Herrera, G. y Ramirez, J. (eds.), América Latina migrante: Estado, familia, identidades. Quito, FLACSO - Ministerio de Cultura, pp. 343-360.

FernÁndez-Kelly, M.P. y GarcíA, A. (1990) "Power surrendered, power restored: the politics of work and family among Hispanic garment workers in California and Florida”, en Tilly, L.A. y Gurin, P. (comps.), Women, politics and changes, New York, Russell-Sage foundation, pp. 130-149.

Herrera, G. (2008) "States, Work, and Social Reproduction through the Lens of Migrant Experience: Ecuadorian Domestic Workers in Madrid" en Bakker, I. y Silvey, R. (eds.) Beyond States and Markets: The Challenges of Social Reproduction. New York, Routledge.

Herrera, G. (2007) “Mujeres ecuatorianas en el trabajo doméstico en España. Estrategias de inclusión y exclusión” en Víctor Breton et al. Ciudadanía y Exclusión: Ecuador-España. Una mirada frente al Espejo. Madrid, Los libros de la Catarata.

Herrera, G. (2004) “Género, familia y migración en el Ecuador. Lo viejo y lo nuevo. En Norma Fuller (ed.) Jerarquías en Jaque. Estudios de género en el área andina. Lima: CLACSO British Council.

Herrera, G. (2006) "Precarización del trabajo, crisis de reproducción social y migración femenina. Ecuatorianas en España y Estados Unidos” en Herrera Gioconda La persistencia de la desigualdad. Género, trabajo y pobreza en América Latina. (ed.) FLACSO,CONAMU, Secretaría Técnica del Frente Social.

Herrera, G. y Martínez, A. (mayo 2002): “Género y migración en la región Sur” Informe de investigación, Quito, FLACSO-Ecuador.

Herrera, G. y CArrillo, M. C. (2009) "Transformaciones familiares en la experiencia migratoria ecuatoriana. Una mirada desde los contextos de salida”, Revue Mélanges de la Casa de Velasquez, 39(1): 97-114.

Hinojosa Gordonava, A. (2009): Buscando la vida: familias bolivianas transnacionales en España, La Paz, CLACSOFundación PIEB. 
Hondagneu-Sotelo, P. y Ávila, E. (2003) "I'm here but I'm there. The Meanings of Latina Transnational Motherhood" en Hondagneu-Sotelo, P. (ed) Gender and U.S. Inmigration. Contemporary Trends, Berkeley-Los Angeles-London, University of California Press. Lagomarsino, F. (2005) "Famiglia e migrazione : un rapporto complesso. Il caso delle famiglie migrantie cuadoriane a Genova”. Ponencia presentada en la Conferencia Internacional: Migraciones, transnacionalismo e identidades: la experiencia ecuatoriana, Quito, Ecuador 15-17 de enero.

LENTZ, C. (1984): Estrategias de Reproducción y migración temporaria. Indígenas de Cajabamba / Chimborazo en Ecuador Debate 8: Migraciones y migrantes, Quito, CAAP.

Martínez, L. (1984): "Migraciones y Cambios en las estrategias Familiares de las Comunidades Indígenas de la Sierra" en Ecuador Debate 8: Migraciones y migrantes, Quito, CAAP.

MisrA, J. et al. (2005): "The Globalization of Carework: Immigration, Economic Restructuring, and the World-System. Ponencia presentada en el seminario "International Conference on Migration and Domestic Work in Global Perspective", Wassenaar, The Netherlands Institute for Advanced Studies, 26 - 29 mayo.

Montero, L. y Poveda, P. (2003) Ser Castañera. Cadena productiva de la industria de la castaña en Riberalta. La Paz, CEDLA.

Oso, L. (2008) "Mujeres latinoamericanas en España y trabajo sexual” en Herrera G. y Ramírez, J. (eds). América Latina migrante: Estado, familia, identidade, Quito, FLACSO - Ministerio de Cultura, pp. 223-241.

Piscitelli, A. (2008) "Industria del sexo y mercado matrimonial: la migración brasileña hacia Italia en el marco del turismo sexual internacional”, en Herrera, G. y Ramirez, J. (eds.), América Latina migrante: Estado, familia, identidades. Quito, FLACSO - Ministerio de Cultura, pp. 179-200.

Pribilsky, J. (2007): La Chulla Vida. Gender, Migration and the Family in Andean Ecuador and New York City: Syracuse University Press.

Reist, D. y Riaño, Y. (2008) "Hablando de aquí y de allá. Patrones de comunicación transnacional entre migrantes y sus familiares" en Herrera,G. y Ramírez, J. (eds.) América Latina migrante: Estado, familia, identidades. Quito, FLACSO - Ministerio de Cultura, pp. 303-324.

Roman, O. (2009): Mientras no estamos. Migración de mujeres madres de Cochabamba a España, Cochabamba, CESU-UMSA.

Rosas, C. (2008) Varones al son de la Migración. Migración Internacional y Masculinidades de Veracruz a Chicago, México, El Colegio de México.

Salazar Parreñas, R. (2001) Servants of Globalization: Women, Migration and Domestic Work, Standford, Standford University Press.

SASsEn, S. (2003) "Strategic Instantiations of Gendering in the Global Economy" en Hondagneu-Sotelo, P. (ed.) Gender and US Inmigration, Contemporary Trends. Los Angeles, University of California Press.

Sorensen, N. (2008) "La familia transnacional de latinoamericanos en Europa” en Herrera, G. y Ramírez, J. (eds.) América Latina migrante: Estado, familia, identidades, Quito, FLACSO - Ministerio de Cultura, pp.259-280.

SweEtman, C. (1998):Gender and migration, Oxford, OXFAM -GB.

WAGNER, H. (2008) 'Maternidad transnacional: discursos, estereotipos, prácticas', en Herrera G., and Ramirez, J. (eds) América Latina Migrante. Estado, familia, identidad. Quito, FLACSO, pp. 325-342.

YeAts, N. (2005) "Global care chains: a critical introduction” en Global Migration perspectives, Geneva, Global Comission on International Migration 44.

Young, B. (2003) "Financial crisis and social reproduction: Asia, Argentina and Brazil en Bakker, I. y Gilí, S. (eds.), Power, production and social reproduction: Human insecurity in the global political economy, New York, Palgrave and MacMillan, pp. 103-123. 